Acta Crystallographica Section E

Structure Reports

Online

ISSN 1600-5368

\section{1-(4-Chlorobenzylideneamino)pyridinum iodide}

\section{Yong-Tao Cui, Jian-Qiang Wang, Chun-Xiang Ji, Hai-Bo Wang and Guo Cheng*}

College of Science, Nanjing University of Technolgy, Xinmofan Road No. 5 Nanjing, Nanjing 210009, People's Republic of China

Correspondence e-mail: guocheng@njut.edu.cn

Received 17 December 2008; accepted 23 December 2008

Key indicators: single-crystal X-ray study; $T=291 \mathrm{~K}$; mean $\sigma(\mathrm{C}-\mathrm{C})=0.007 \AA$; $R$ factor $=0.027 ; w R$ factor $=0.093 ;$ data-to-parameter ratio $=15.2$.

In the title compound, $\mathrm{C}_{12} \mathrm{H}_{10} \mathrm{ClN}_{2}^{+} \cdot \mathrm{I}^{-}$, the aromatic rings are oriented at a dihedral angle of $54.55(3)^{\circ}$. In the crystal structure, intermolecular $\mathrm{C}-\mathrm{H} \cdots \mathrm{I}$ and $\mathrm{C}-\mathrm{H} \cdots \mathrm{Cl}$ hydrogen bonds link the molecules.

\section{Related literature}

For background, see: Okamoto et al. (1967). For bond-length data, see: Allen et al. (1987).

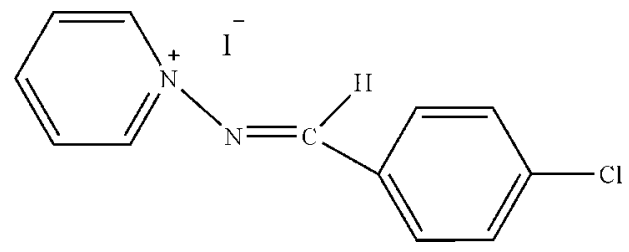

\section{Experimental}

\section{Crystal data}

$\mathrm{C}_{12} \mathrm{H}_{10} \mathrm{ClN}_{2}^{+} \cdot \mathrm{I}^{-}$
$M_{r}=344.57$
Triclinic, $P \overline{1}$
$a=6.5105(14) \AA$
$b=7.1748(15) \AA$
$c=14.223(3) \AA$

$\mu=2.72 \mathrm{~mm}^{-1}$

$T=291(2) \mathrm{K}$

Data collection

Enraf-Nonius CAD-4 diffractometer

Absorption correction: $\psi$ scan (North et al., 1968)

$T_{\min }=0.772, T_{\max }=0.812$

3220 measured reflections

Refinement

$R\left[F^{2}>2 \sigma\left(F^{2}\right)\right]=0.027$

$w R\left(F^{2}\right)=0.093$

$S=1.05$

2205 reflections
$0.10 \times 0.10 \times 0.08 \mathrm{~mm}$

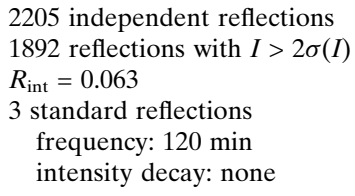

145 parameters

$\mathrm{H}$-atom parameters constrained

$\Delta \rho_{\max }=0.53{\mathrm{e} \AA^{-3}}^{-3}$

$\Delta \rho_{\text {min }}=-0.63{\mathrm{e} \AA^{-3}}^{-3}$

Table 1

Hydrogen-bond geometry $\left(\AA{ }^{\circ}\right)$.

\begin{tabular}{lllll}
\hline$D-\mathrm{H} \cdots A$ & $D-\mathrm{H}$ & $\mathrm{H} \cdots A$ & $D \cdots A$ & $D-\mathrm{H} \cdots A$ \\
\hline $\mathrm{C} 1-\mathrm{H} 1 \cdots \mathrm{I} 1$ & 0.93 & 3.04 & $3.857(5)$ & 147 \\
$\mathrm{C} 5-\mathrm{H} 5 \cdots \mathrm{Cl} 1^{\mathrm{i}}$ & 0.93 & 2.79 & $3.691(6)$ & 162 \\
\hline
\end{tabular}

Symmetry code: (i) $-x,-y,-z+1$.

Data collection: CAD-4 Software (Enraf-Nonius, 1989); cell refinement: CAD-4 Software; data reduction: XCAD4 (Harms \& Wocadlo, 1995); program(s) used to solve structure: SHELXS97 (Sheldrick, 2008); program(s) used to refine structure: SHELXL97 (Sheldrick, 2008); molecular graphics: ORTEP-3 for Windows (Farrugia, 1997); software used to prepare material for publication: SHELXL97.

The authors thank the Center of Testing and Analysis, Nanjing University, for support.

Supplementary data and figures for this paper are available from the IUCr electronic archives (Reference: HK2600).

\title{
References
}

Allen, F. H., Kennard, O., Watson, D. G., Brammer, L., Orpen, A. G. \& Taylor, R. (1987). J. Chem. Soc. Perkin Trans. 2, pp. S1-19.

Enraf-Nonius (1989). CAD-4 Software. Enraf-Nonius, Delft. The Netherlands.

Farrugia, L. J. (1997). J. Appl. Cryst. 30, 565.

Harms, K. \& Wocadlo, S. (1995). XCAD4. University of Marburg, Germany.

North, A. C. T., Phillips, D. C. \& Mathews, F. S. (1968). Acta Cryst. A24, 351359.

Okamoto, T., Hirobe, M., Sato, R. (1967). Yakugaku Zasshi, 87, 994-996.

Sheldrick, G. M. (2008). Acta Cryst. A64, 112-122. 


\section{supporting information}

Acta Cryst. (2009). E65, o228 [doi:10.1107/S1600536808043729]

\section{1-(4-Chlorobenzylideneamino)pyridinum iodide}

\section{Yong-Tao Cui, Jian-Qiang Wang, Chun-Xiang Ji, Hai-Bo Wang and Guo Cheng}

\section{S1. Comment}

Some derivatives of 1-aminopyidium iodide are important chemical materials. We report herein the crystal structure of the title compound.

In the molecule of the title compound (Fig. 1), the bond lengths (Allen et al., 1987) and angles are within normal ranges. Rings A (N1/C1-C5) and B (C7-C12) are, of course, planar, and they are oriented at a dihedral angle of 54.55 (3).

In the crystal structure, intramolecular $\mathrm{C}-\mathrm{H} \cdots \mathrm{I}$ and intermolecular $\mathrm{C}-\mathrm{H} \cdots \mathrm{Cl}$ hydrogen bonds (Table 1) link the molecules (Fig. 2), in which they may be effective in the stabilization of the structure. The $\pi-\pi$ contacts between the pyridine rings and the benzene rings, $\mathrm{Cg} 1-\mathrm{Cg} 1^{\mathrm{i}}$ and $\mathrm{Cg} 2-\mathrm{Cg} 2^{\mathrm{ii}}$ [symmetry codes: (i) $1-\mathrm{x},-\mathrm{y},-\mathrm{z}$; (ii) $1-\mathrm{x},-\mathrm{y}, 1-\mathrm{z}$, where $\mathrm{Cg} 1$ and $\mathrm{Cg} 2$ are centroids of the rings $\mathrm{A}(\mathrm{N} 1 / \mathrm{C} 1-\mathrm{C} 5)$ and $\mathrm{B}(\mathrm{C} 7-\mathrm{C} 12)$, respectively] may further stabilize the structure, with centroid-centroid distances of 4.130 (3) $\AA$ and 4.056 (3) $\AA$.

\section{S2. Experimental}

For the preparation of the title compound, 1-aminopyridinium iodide $(22.2 \mathrm{~g}, 0.10 \mathrm{~mol})$ was dissolved in ethanol $(20 \mathrm{ml})$, 4-methylbenzaldehyde ( $12.4 \mathrm{~g}, 0.1 \mathrm{~mol})$ was added with stirring, and then the mixture was heated at reflux for $5 \mathrm{~h}$. Upon cooling to room temperature, a precipitate formed, which was collected by filtration and washed with cold ethanol ( $2 \mathrm{X}$ $10 \mathrm{ml}$ ) to obtain a yellow solid (yield; $24.0 \mathrm{~g}, 70 \%$ ). Crystals suitable for X-ray analysis were obtained by slow evaporation of an ethanol solution.

\section{S3. Refinement}

$\mathrm{H}$ atoms were positioned geometrically, with $\mathrm{C}-\mathrm{H}=0.93 \AA$ for aromatic and methine $\mathrm{H}$ and constrained to ride on their parent atoms, with $\mathrm{U}_{\text {iso }}(\mathrm{H})=1.2 \mathrm{U}_{\mathrm{eq}}(\mathrm{C})$. 


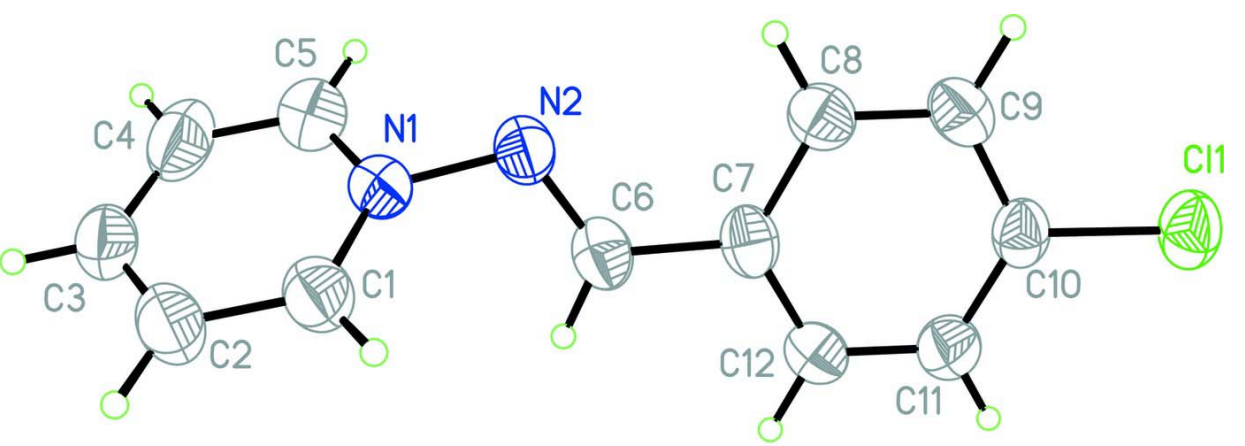

\section{Figure 1}

The molecular structure of the title molecule, with the atom-numbering scheme. Displacement ellipsoids are drawn at the $50 \%$ probability level.

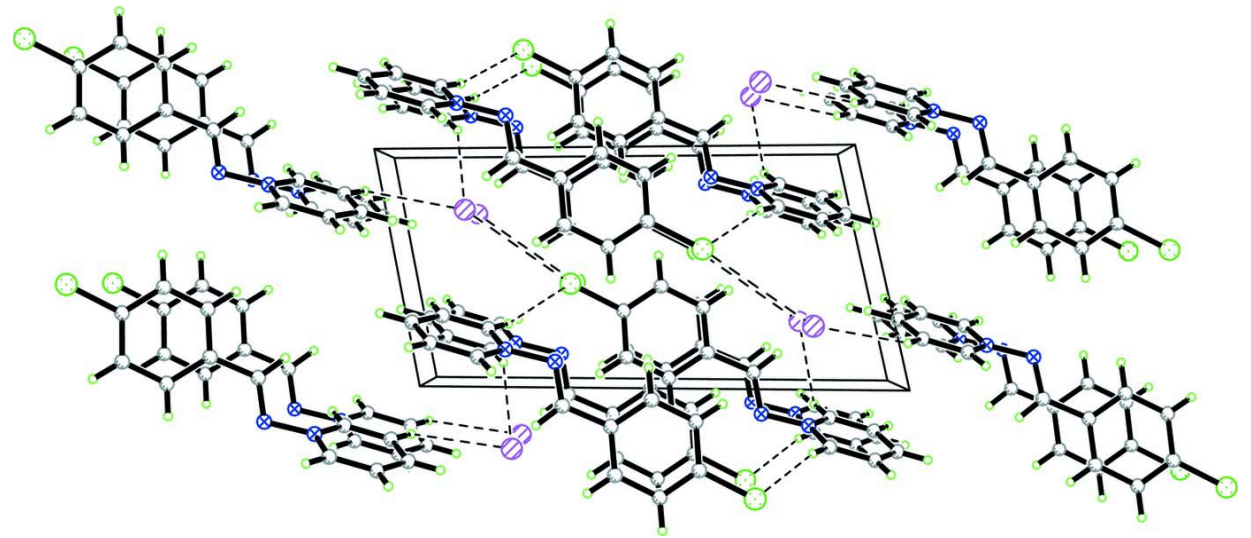

\section{Figure 2}

A partial packing diagram of the title compound. Hydrogen bonds are shown as dashed lines.

\section{1-(4-Chlorobenzylideneamino)pyridinum iodide}

\section{Crystal data}

$\mathrm{C}_{12} \mathrm{H}_{10} \mathrm{ClN}_{2}^{+} \cdot \mathrm{I}^{-}$

$M_{r}=344.57$

Triclinic, $P \overline{1}$

Hall symbol: -P 1

$a=6.5105$ (14) $\AA$

$b=7.1748(15) \AA$

$c=14.223(3) \AA$

$\alpha=76.893(3)^{\circ}$

$\beta=79.183(3)^{\circ}$

$\gamma=80.753(3)^{\circ}$

$V=630.7(2) \AA^{3}$
$Z=2$

$F(000)=332$

$D_{\mathrm{x}}=1.814 \mathrm{Mg} \mathrm{m}^{-3}$

Mo $K \alpha$ radiation, $\lambda=0.71073 \AA$

Cell parameters from 25 reflections

$\theta=2.1-25.3^{\circ}$

$\mu=2.72 \mathrm{~mm}^{-1}$

$T=291 \mathrm{~K}$

Block, yellow

$0.10 \times 0.10 \times 0.08 \mathrm{~mm}$ 


\section{Data collection}

Enraf-Nonius CAD-4 diffractometer

Radiation source: fine-focus sealed tube Graphite monochromator $\omega / 2 \theta$ scans

Absorption correction: $\psi$ scan

(North et al., 1968)

$T_{\min }=0.772, T_{\max }=0.812$

3220 measured reflections

\section{Refinement}

Refinement on $F^{2}$

Least-squares matrix: full

$R\left[F^{2}>2 \sigma\left(F^{2}\right)\right]=0.027$

$w R\left(F^{2}\right)=0.093$

$S=1.05$

2205 reflections

145 parameters

0 restraints

Primary atom site location: structure-invariant direct methods
2205 independent reflections

1892 reflections with $I>2 \sigma(I)$

$R_{\text {int }}=0.063$

$\theta_{\max }=25.0^{\circ}, \theta_{\min }=2.9^{\circ}$

$h=-7 \rightarrow 7$

$k=-8 \rightarrow 8$

$l=-15 \rightarrow 16$

3 standard reflections every $120 \mathrm{~min}$ intensity decay: none

Secondary atom site location: difference Fourier map

Hydrogen site location: inferred from neighbouring sites

$\mathrm{H}$-atom parameters constrained $w=1 /\left[\sigma^{2}\left(F_{\mathrm{o}}^{2}\right)+(0.0479 P)^{2}\right]$ where $P=\left(F_{\mathrm{o}}^{2}+2 F_{\mathrm{c}}{ }^{2}\right) / 3$

$(\Delta / \sigma)_{\max }<0.001$

$\Delta \rho_{\max }=0.53 \mathrm{e}^{-3}$

$\Delta \rho_{\min }=-0.63$ e $\AA^{-3}$

\section{Special details}

Geometry. All e.s.d.'s (except the e.s.d. in the dihedral angle between two 1.s. planes) are estimated using the full covariance matrix. The cell e.s.d.'s are taken into account individually in the estimation of e.s.d.'s in distances, angles and torsion angles; correlations between e.s.d.'s in cell parameters are only used when they are defined by crystal symmetry. An approximate (isotropic) treatment of cell e.s.d.'s is used for estimating e.s.d.'s involving 1.s. planes.

Refinement. Refinement of $F^{2}$ against ALL reflections. The weighted $R$-factor $w R$ and goodness of fit $S$ are based on $F^{2}$, conventional $R$-factors $R$ are based on $F$, with $F$ set to zero for negative $F^{2}$. The threshold expression of $F^{2}>\sigma\left(F^{2}\right)$ is used only for calculating $R$-factors $(\mathrm{gt})$ etc. and is not relevant to the choice of reflections for refinement. $R$-factors based on $F^{2}$ are statistically about twice as large as those based on $F$, and $R$-factors based on ALL data will be even larger.

Fractional atomic coordinates and isotropic or equivalent isotropic displacement parameters $\left(\AA^{2}\right)$

\begin{tabular}{lllll}
\hline & $x$ & $y$ & $z$ & $U_{\text {iso }} * / U_{\text {eq }}$ \\
\hline I1 & $0.91966(5)$ & $0.25912(5)$ & $0.16564(2)$ & $0.05410(17)$ \\
C11 & $0.1793(2)$ & $0.4285(2)$ & $0.64323(10)$ & $0.0562(3)$ \\
N1 & $0.4087(6)$ & $-0.1676(5)$ & $0.2056(3)$ & $0.0410(9)$ \\
N2 & $0.3499(6)$ & $-0.1179(6)$ & $0.3000(3)$ & $0.0434(9)$ \\
C1 & $0.6038(8)$ & $-0.1437(7)$ & $0.1537(4)$ & $0.0495(12)$ \\
H1 & 0.7003 & -0.0912 & 0.1775 & $0.059^{*}$ \\
C2 & $0.6541(10)$ & $-0.1996(7)$ & $0.0648(4)$ & $0.0608(15)$ \\
H2 & 0.7863 & -0.1852 & 0.0274 & $0.073 *$ \\
C3 & $0.5076(10)$ & $-0.2770(7)$ & $0.0313(4)$ & $0.0585(14)$ \\
H3 & 0.5404 & -0.3131 & -0.0291 & $0.070^{*}$ \\
C4 & $0.3107(11)$ & $-0.3012(8)$ & $0.0878(4)$ & $0.0654(16)$ \\
H4 & 0.2111 & -0.3533 & 0.0658 & $0.079 *$ \\
C5 & $0.2669(9)$ & $-0.2469(7)$ & $0.1763(4)$ & $0.0562(13)$ \\
H5 & 0.1380 & -0.2654 & 0.2160 & $0.067^{*}$ \\
C6 & $0.3740(8)$ & $0.0561(7)$ & $0.2976(3)$ & $0.0451(11)$
\end{tabular}




\begin{tabular}{lllll} 
H6 & 0.4240 & 0.1326 & 0.2382 & $0.054^{*}$ \\
C7 & $0.3254(7)$ & $0.1391(7)$ & $0.3855(3)$ & $0.0415(11)$ \\
C8 & $0.2488(7)$ & $0.0346(7)$ & $0.4776(3)$ & $0.0442(11)$ \\
H8 & 0.2289 & -0.0937 & 0.4851 & $0.053^{*}$ \\
C9 & $0.2026(8)$ & $0.1216(7)$ & $0.5574(3)$ & $0.0474(12)$ \\
H9 & 0.1510 & 0.0536 & 0.6189 & $0.057^{*}$ \\
C10 & $0.2355(7)$ & $0.3145(7)$ & $0.5436(3)$ & $0.0424(11)$ \\
C11 & $0.3049(8)$ & $0.4181(7)$ & $0.4539(3)$ & $0.0479(12)$ \\
H11 & 0.3202 & 0.5476 & 0.4461 & $0.058^{*}$ \\
C12 & $0.3526(8)$ & $0.3297(7)$ & $0.3745(3)$ & $0.0460(11)$ \\
H12 & 0.4033 & 0.3992 & 0.3132 & $0.055^{*}$ \\
\hline
\end{tabular}

Atomic displacement parameters $\left(\AA^{2}\right)$

\begin{tabular}{lllllll}
\hline & $U^{11}$ & $U^{22}$ & $U^{33}$ & $U^{12}$ & $U^{13}$ & $U^{23}$ \\
\hline $\mathrm{I} 1$ & $0.0580(3)$ & $0.0587(3)$ & $0.0457(2)$ & $-0.01153(17)$ & $-0.00263(16)$ & $-0.01233(16)$ \\
C11 & $0.0519(8)$ & $0.0723(9)$ & $0.0497(7)$ & $-0.0091(6)$ & $-0.0010(6)$ & $-0.0280(6)$ \\
N1 & $0.041(2)$ & $0.043(2)$ & $0.039(2)$ & $-0.0075(18)$ & $-0.0030(18)$ & $-0.0090(17)$ \\
N2 & $0.045(2)$ & $0.048(2)$ & $0.038(2)$ & $-0.0064(18)$ & $-0.0026(17)$ & $-0.0132(17)$ \\
C1 & $0.055(3)$ & $0.048(3)$ & $0.043(3)$ & $-0.004(2)$ & $-0.004(2)$ & $-0.009(2)$ \\
C2 & $0.076(4)$ & $0.050(3)$ & $0.046(3)$ & $0.001(3)$ & $0.009(3)$ & $-0.010(2)$ \\
C3 & $0.083(4)$ & $0.047(3)$ & $0.044(3)$ & $-0.009(3)$ & $0.000(3)$ & $-0.016(2)$ \\
C4 & $0.094(5)$ & $0.050(3)$ & $0.061(4)$ & $-0.006(3)$ & $-0.023(3)$ & $-0.021(3)$ \\
C5 & $0.060(4)$ & $0.055(3)$ & $0.055(3)$ & $-0.009(3)$ & $-0.008(3)$ & $-0.014(3)$ \\
C6 & $0.050(3)$ & $0.049(3)$ & $0.036(3)$ & $0.001(2)$ & $-0.008(2)$ & $-0.010(2)$ \\
C7 & $0.037(3)$ & $0.050(3)$ & $0.038(3)$ & $0.003(2)$ & $-0.008(2)$ & $-0.014(2)$ \\
C8 & $0.041(3)$ & $0.046(3)$ & $0.043(3)$ & $-0.004(2)$ & $-0.003(2)$ & $-0.009(2)$ \\
C9 & $0.047(3)$ & $0.056(3)$ & $0.036(3)$ & $-0.007(2)$ & $0.000(2)$ & $-0.008(2)$ \\
C10 & $0.034(3)$ & $0.056(3)$ & $0.041(3)$ & $-0.006(2)$ & $-0.006(2)$ & $-0.017(2)$ \\
C11 & $0.055(3)$ & $0.044(3)$ & $0.046(3)$ & $-0.008(2)$ & $-0.009(2)$ & $-0.009(2)$ \\
C12 & $0.052(3)$ & $0.047(3)$ & $0.037(3)$ & $-0.010(2)$ & $-0.006(2)$ & $-0.003(2)$ \\
& & & & & & \\
\hline
\end{tabular}

Geometric parameters $\left(A,{ }^{\circ}\right)$

\begin{tabular}{llll}
\hline $\mathrm{C} 11-\mathrm{C} 10$ & $1.746(5)$ & $\mathrm{C} 5-\mathrm{H} 5$ & 0.9300 \\
$\mathrm{~N} 1-\mathrm{C} 5$ & $1.331(7)$ & $\mathrm{C} 6-\mathrm{C} 7$ & $1.465(6)$ \\
$\mathrm{N} 1-\mathrm{C} 1$ & $1.359(6)$ & $\mathrm{C} 6-\mathrm{H} 6$ & 0.9300 \\
$\mathrm{~N} 1-\mathrm{N} 2$ & $1.434(5)$ & $\mathrm{C} 7-\mathrm{C} 12$ & $1.377(6)$ \\
$\mathrm{N} 2-\mathrm{C} 6$ & $1.275(6)$ & $\mathrm{C} 7-\mathrm{C} 8$ & $1.399(6)$ \\
$\mathrm{C} 1-\mathrm{C} 2$ & $1.379(7)$ & $\mathrm{C} 8-\mathrm{C} 9$ & $1.378(6)$ \\
$\mathrm{C} 1-\mathrm{H} 1$ & 0.9300 & $\mathrm{C} 8-\mathrm{H} 8$ & 0.9300 \\
$\mathrm{C} 2-\mathrm{C} 3$ & $1.380(8)$ & $\mathrm{C} 9-\mathrm{C} 10$ & $1.399(7)$ \\
$\mathrm{C} 2-\mathrm{H} 2$ & 0.9300 & $\mathrm{C} 9-\mathrm{H} 9$ & 0.9300 \\
$\mathrm{C} 3-\mathrm{C} 4$ & $1.393(9)$ & $\mathrm{C} 10-\mathrm{C} 11$ & $1.358(7)$ \\
$\mathrm{C} 3-\mathrm{H} 3$ & 0.9300 & $\mathrm{C} 11-\mathrm{C} 12$ & $1.380(6)$ \\
$\mathrm{C} 4-\mathrm{C} 5$ & $1.367(7)$ & $\mathrm{C} 11-\mathrm{H} 11$ & 0.9300 \\
$\mathrm{C} 4-\mathrm{H} 4$ & 0.9300 & $\mathrm{C} 12-\mathrm{H} 12$ & 0.9300
\end{tabular}




$\begin{array}{ll}\mathrm{C} 5-\mathrm{N} 1-\mathrm{C} 1 & 123.4(4) \\ \mathrm{C} 5-\mathrm{N} 1-\mathrm{N} 2 & 116.0(4) \\ \mathrm{C} 1-\mathrm{N} 1-\mathrm{N} 2 & 120.4(4) \\ \mathrm{C} 6-\mathrm{N} 2-\mathrm{N} 1 & 112.2(4) \\ \mathrm{N} 1-\mathrm{C} 1-\mathrm{C} 2 & 118.0(5) \\ \mathrm{N} 1-\mathrm{C} 1-\mathrm{H} 1 & 121.0 \\ \mathrm{C} 2-\mathrm{C} 1-\mathrm{H} 1 & 121.0 \\ \mathrm{C} 1-\mathrm{C} 2-\mathrm{C} 3 & 119.7(5) \\ \mathrm{C} 1-\mathrm{C} 2-\mathrm{H} 2 & 120.1 \\ \mathrm{C} 3-\mathrm{C} 2-\mathrm{H} 2 & 120.1 \\ \mathrm{C} 2-\mathrm{C} 3-\mathrm{C} 4 & 120.2(5) \\ \mathrm{C} 2-\mathrm{C} 3-\mathrm{H} 3 & 119.9 \\ \mathrm{C} 4-\mathrm{C} 3-\mathrm{H} 3 & 119.9 \\ \mathrm{C} 5-\mathrm{C} 4-\mathrm{C} 3 & 118.6(5) \\ \mathrm{C} 5-\mathrm{C} 4-\mathrm{H} 4 & 120.7 \\ \mathrm{C} 3-\mathrm{C} 4-\mathrm{H} 4 & 120.7 \\ \mathrm{~N} 1-\mathrm{C} 5-\mathrm{C} 4 & 120.0(5) \\ \mathrm{N} 1-\mathrm{C} 5-\mathrm{H} 5 & 120.0 \\ \mathrm{C} 4-\mathrm{C} 5-\mathrm{H} 5 & 120.0 \\ \mathrm{~N} 2-\mathrm{C} 6-\mathrm{C} 7 & 122.0(4) \\ \end{array}$

N2- C6- 66

$\mathrm{C} 7-\mathrm{C} 6-\mathrm{H} 6$

$\mathrm{C} 12-\mathrm{C} 7-\mathrm{C} 8$

$\mathrm{C} 12-\mathrm{C} 7-\mathrm{C} 6$

$\mathrm{C} 8-\mathrm{C} 7-\mathrm{C} 6$

$\mathrm{C} 9-\mathrm{C} 8-\mathrm{C} 7$

$\mathrm{C} 9-\mathrm{C} 8-\mathrm{H} 8$

$\mathrm{C} 7-\mathrm{C} 8-\mathrm{H} 8$

$\mathrm{C} 8-\mathrm{C} 9-\mathrm{C} 10$

$\mathrm{C} 8-\mathrm{C} 9-\mathrm{H} 9$

$\mathrm{C} 10-\mathrm{C} 9-\mathrm{H} 9$

$\mathrm{C} 11-\mathrm{C} 10-\mathrm{C} 9$

$\mathrm{C} 11-\mathrm{C} 10-\mathrm{Cl}$

C9-C10-Cl1

$\mathrm{C} 10-\mathrm{C} 11-\mathrm{C} 12$

$\mathrm{C} 10-\mathrm{C} 11-\mathrm{H} 11$

C12-C11-H11

$\mathrm{C} 7-\mathrm{C} 12-\mathrm{C} 11$

$\mathrm{C} 7-\mathrm{C} 12-\mathrm{H} 12$

$\mathrm{C} 11-\mathrm{C} 12-\mathrm{H} 12$
119.0

119.0

$119.8(4)$

$117.4(4)$

$122.8(4)$

$120.1(4)$

119.9

119.9

118.4 (4)

120.8

120.8

$121.8(4)$

$118.7(4)$

$119.5(4)$

$119.5(4)$

120.2

120.2

120.4 (4)

119.8

119.8

Hydrogen-bond geometry $\left(\AA,{ }^{\circ}\right)$

\begin{tabular}{lllll}
\hline$D-\mathrm{H} \cdots A$ & $D-\mathrm{H}$ & $\mathrm{H} \cdots A$ & $D \cdots A$ & $D-\mathrm{H} \cdots A$ \\
\hline $\mathrm{C} 1-\mathrm{H} 1 \cdots \mathrm{I} 1$ & 0.93 & 3.04 & $3.857(5)$ & 147 \\
$\mathrm{C} 5-\mathrm{H} 5 \cdots \mathrm{C} 11^{\mathrm{i}}$ & 0.93 & 2.79 & $3.691(6)$ & 162 \\
\hline
\end{tabular}

Symmetry code: (i) $-x,-y,-z+1$. 